\title{
Why does the Dyad-4PNO model of Kern and Culpepper (2020) fit real data?
}

\author{
Gunter Maris \\ ACT, University of Amsterdam \\ Timo Bechger \\ $\mathrm{ACT}$ \\ Benjamin Deonovic \\ $\mathrm{ACT}$
}

\begin{abstract}
This note aims to elucidate why the Dyad-4PNO model of Kern and Culpepper (2020) can be expected to fit real data reasonably well. The main result is that the Dyad-4PNO approximates a latent tree model. We offer a simple proof of identifiability, and draw some implications for psychological measurement in practice.
\end{abstract}

\section{Introduction}

Kern and Culpepper (2020) introduce the Dyad-4PNO as a variation on the four parameter normal ogive (4PNO) model of Barton and Lord (1981). Using results from Gu and $\mathrm{Xu}$ (2018), they show that the parameters of the Dyad-4PNO are identifiable which is quite remarkable given that the identifiability of the three parameter logistic model (3PL) has not been completely settled (e.g., Maris and Bechger (2009)). Although the results of Kern and Culpepper (2020) are mathematically correct, the question remains why one would expect the Dyad-4PNO to fit real data satisfactorily.

We shortly review the Dyad-4PNO model, explain why this model can be expected to do a reasonable job at fitting real data, and discuss practical implications for measurement in psychology. In passing, we will obtain results on parameter identifiability in a way that is much simpler than $\mathrm{Gu}$ and $\mathrm{Xu}(2018)$.

\section{The Dyad-4PNO model}

The $4 \mathrm{PNO}$ model is characterized by the following item response function (IRF):

$$
P\left(Y_{j}=1 \mid \theta, \alpha_{j}, \beta_{j}, g_{j}, s_{j}\right)=g_{j}+\left(1-s_{j}-g_{j}\right) \Psi\left(\alpha_{j} \theta-\beta_{j}\right)
$$

in which $\Psi$ denotes the normal ogive. The model is a mixture model. If we introduce an augmented binary variable, $W_{j}$, distributed as a 2 PNO model:

$$
P\left(W_{j}=1 \mid \theta, \alpha_{j}, \beta_{j}\right)=\Psi\left(\alpha_{j} \theta-\beta_{j}\right)
$$


and define the response probability conditionally on $W_{j}$ as follows:

$$
p\left(Y_{j}=1 \mid W_{j}=w_{j}, g_{j}, s_{j}\right)=\left(1-s_{j}\right)^{w_{j}} g_{j}^{1-w_{j}}
$$

we recover the $4 \mathrm{PNO}$ for the item responses.

The Dyad-4PNO differs from the $4 \mathrm{PNO}$ in that the augmented variable is shared between pairs of items. That is, if item $j$ and item $k$ form a dyad, then $W_{j}=W_{k}$ with probability one. As Kern and Culpepper (2020) note in their discussion, the essential property of the model is that items can be grouped such that for any two items belonging to the same group, the augmented variables are the same (with probability one), and every group consists of at least two items.

Through the connection of the $4 \mathrm{PNO}$ and the Dyad-4PNO to the higher order DINA model of De La Torre and Douglas (2004), Kern and Culpepper (2020) are able to make use of the results of $\mathrm{Gu}$ and $\mathrm{Xu}$ (2018) to demonstrate that in the Dyad-4PNO parameters are identifiable. Being able to demonstrate parameter identifiability for any statistical model is essential, to ensure consistent parameter estimates, for instance, but are the restrictions on the augmented variables, imposed by the Dyad-4PNO for identifiability, commensurate with real response behavior, and if so why?

\section{The Dyad-4PNO model as an approximation}

In this section we demonstrate that from a latent tree model for the augmented variables we directly obtain the Dyad-4PL, and its cousin the Dyad-4PNO, as a rank one approximation.

\section{Latent tree models}

Latent tree models are a subclass of graphical models in which any two variables, observed or augmented, (called nodes) are connected via a unique path. The left panel in Figure 1 shows an example. The squares in Figure 1 correspond with observed responses and the circles with augmented variables. Edges between nodes correspond with a bivariate distribution of the nodes at both ends of the edge. Two nodes that are connected via one intermediate node are called siblings, and a node that is only connected to one other node is called a leaf node. For notational convenience, we index nodes from left to right and bottom to top, such that we can refer to the indices in equations.

Tree models encode hierarchical structure and are useful in education where many assessment frameworks are hierarchical. For instance, the ACT holistic framework of Camara, O'connor, Mattern, and Hanson (2015) is constructed as a tree of skills. For our present purpose, we let the augmented variables correspond to skills and assume that these are either mastered or not mastered. Every observed response $Y_{j}$ is connected to one lowest level skill $W_{k}$ (the duplication of notation is intentional) and we assume that, conditional on $W_{k}$, the distribution for $Y_{j}$ is the same as in the $4 \mathrm{PNO}$ and Dyad-4PNO; i.e., Equation 3 .

A latent tree model (e.g., Wainwright and Jordan (2008, p. 77)) has the following 


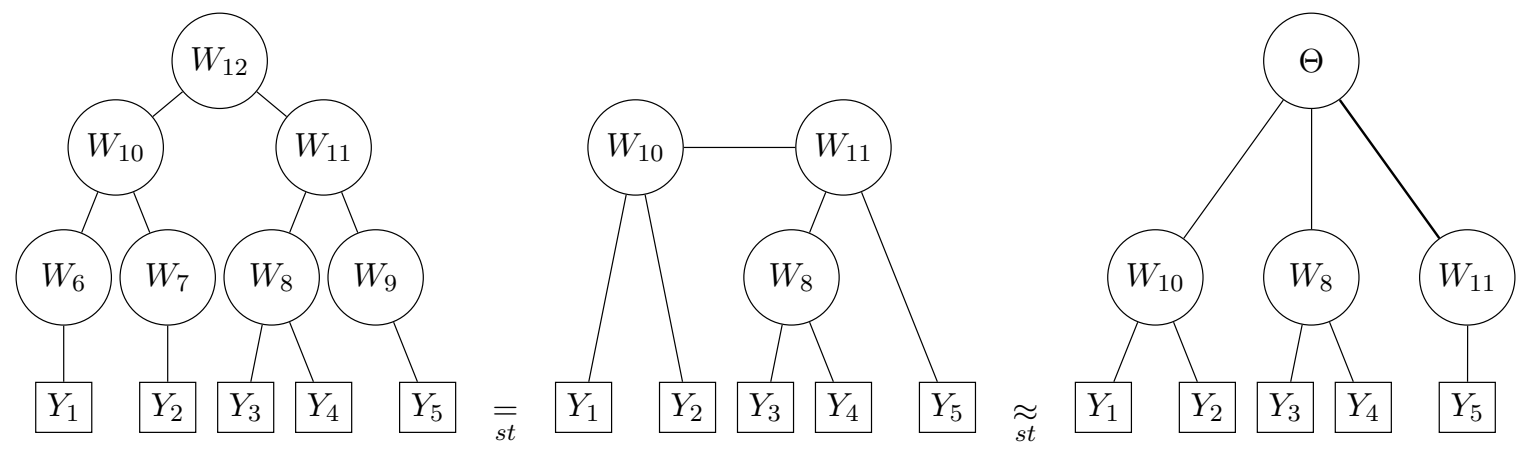

Figure 1. Unidentified latent tree model (left), its implied identified latent tree model (middle), and its rank one approximation (right). $\overline{\overline{s t}}$ and $\underset{s t}{\approx}$ denote that the observed variables $\mathbf{Y}$ are exactly or approximately identically distributed.

equivalent representations:

$$
\begin{aligned}
p(\mathbf{w}) & =\frac{\exp \left(-\mathbf{w}^{\top} \boldsymbol{\beta}+\mathbf{w}^{\top} \boldsymbol{\Sigma} \mathbf{w}\right)}{Z} \\
& =\prod_{j} \pi_{j}\left(w_{j}\right) \prod_{<j k>} \frac{\pi_{j k}\left(w_{j}, w_{k}\right)}{\pi_{j}\left(w_{j}\right) \pi_{k}\left(w_{k}\right)},
\end{aligned}
$$

where the matrix $\boldsymbol{\Sigma}$ encodes the adjacency matrix of the tree (i.e., a zero entry means that the corresponding nodes are not neighbors), $\boldsymbol{\beta}$ are main effects, $Z$ ensures that the distribution sums to one, $\pi_{j}$ is the univariate marginal of $W_{j}$, and $\pi_{j k}$ is the bivariate marginal of two augmented variables that are directly connected (denoted by $<j k>$ ). We use $\boldsymbol{\pi}_{k j}$ to denote the distribution of $W_{k}$ (row) and $W_{j}$ (column), $\boldsymbol{\pi}_{k \mid j}$ the corresponding conditional distribution, and $\boldsymbol{\pi}_{k}$ the marginal.

Three things are worth noting. First, as Equation 3 comprises of two conditional distributions, just like $\boldsymbol{\pi}_{k \mid j}$, we find that both $p(\mathbf{w})$ and $p(\mathbf{y}, \mathbf{w})$ are graphical tree models. Second, by directly summing out leaf nodes in $p(\mathbf{y}, \mathbf{w})$ we see that graphical tree models are closed under marginalization with respect to leaf nodes. That is

$$
p(\mathbf{w})=\sum_{\mathbf{y}} p(\mathbf{y}, \mathbf{w}) .
$$

Third, Equation 5 implies that all bivariate marginal distributions (not just those between nodes that are directly connected) can be written in closed form. For example, in the left panel of Figure 1, nodes 6 and 8 are connected via the (unique) path $6-10-12-11-8$ and we directly find the expression for $\boldsymbol{\pi}_{86}$ to be:

$$
\boldsymbol{\pi}_{86}=\boldsymbol{\pi}_{8 \mid 11} \boldsymbol{\pi}_{11 \mid 12} \boldsymbol{\pi}_{12 \mid 10} \boldsymbol{\pi}_{10 \mid 6} \operatorname{diag}\left(\boldsymbol{\pi}_{6}\right),
$$

Hence, in a latent tree model all univariate and bivariate marginals of the observed variables have closed-form expressions.

\section{Rank one approximation to a latent tree model}

The connection between latent tree models and IRT models is a special case of the connection between binary graphical models and IRT (e.g., Epskamp, Maris, Waldorp, and 
Borsboom (2016), Kruis and Maris (2016), Marsman et al. (2018)). The main result is that any binary graphical model can be written as a marginal multi-dimensional logistic IRT model (MIRT). Specifically,

$$
\begin{aligned}
p(\mathbf{w}) & =\frac{\exp \left(-\mathbf{w}^{\boldsymbol{\top}} \boldsymbol{\beta}+\mathbf{w}^{\boldsymbol{\top}} \boldsymbol{\Sigma} \mathbf{w}\right)}{Z} \\
& =\int_{\mathcal{R}^{d}} \prod_{j} \frac{\exp \left(w_{j}\left(-\beta_{j}+2 \sum_{k} \sqrt{\lambda_{k}} \alpha_{j k} \theta_{k}\right)\right)}{1+\exp \left(-\beta_{j}+2 \sum_{k} \sqrt{\lambda_{k}} \alpha_{j k} \theta_{k}\right)} \\
& \times \frac{\prod_{j}\left(1+\exp \left(-\beta_{j}+2 \sum_{k} \sqrt{\lambda_{k}} \alpha_{j k} \theta_{k}\right)\right) \exp \left(-\boldsymbol{\theta}^{\boldsymbol{\top}} \boldsymbol{\theta}\right)}{Z} d \boldsymbol{\theta},
\end{aligned}
$$

where $\lambda_{k}$ is the $k$-th eigenvalue of $\boldsymbol{\Sigma}$, and $\alpha_{j k}$ is the $j$-th element of the $k$-th eigenvector. It is important to observe that in the MIRT formulation, the augmented variables $\mathbf{W}$ are independent conditionally on $\boldsymbol{\Theta}$. Hence, we can integrate out all the augmented variables, except for the leaf nodes, to obtain a MIRT representation of the distribution for the leaf nodes alone. Note that the MIRT formulation comes with a particular distribution of $\Theta$ (Equation 10).

Based on a celebrated Theorem by Eckart and Young (1936) we can approximate (in a least-squares sense) the adjacency matrix $\boldsymbol{\Sigma}$ by a low rank matrix. A rank 3 approximation, for example, is obtained if we keep only the three first eigenvectors and eigenvalues. Marsman, Maris, Bechger, and Glas (2015) use this result to obtain a low rank MIRT approximation to $p(\mathbf{w})$. Taking a rank one approximation, we end up with a $2 \mathrm{PL}$ for $\mathbf{W}$, and hence with a Dyad-4PL for $\mathbf{Y}$. The right-hand panel of Figure 1 shows the approximation to $p(\mathbf{w})$, and Figure 2 the corresponding distribution of the latent variable in Equation 10 for different values of $\lambda_{1}$. The distribution of the latent variable is close to a mixture of two normal distributions, and can be symmetric, skew, or bimodal depending on the magnitude of the eigenvalue. Note that since the joint distribution of $\mathbf{Y}$ and $\mathbf{W}$ is also a binary graphical model, we can also approximate the marginal distribution of $\mathbf{Y}$ with a $2 \mathrm{PL}$. It is clear that the former approximation is going to be the better one, as it captures the tagging of observed responses to augmented variables exactly.

How good can we expect the approximation to be? A key insight derives from the eigenvalue decomposition of a bipartite graph. A bipartite graph comprises of two layers of nodes such that there are only edges between nodes from different layers. A tree graph is a special case of a bipartite graph, with one layer comprising of the odd numbered levels in the tree, and the other of the even numbered levels. It is well known that the eigenvalues of a bipartite graph are symmetrical about zero. Hence, the best you can get is a rank two adjacency matrix with one positive and one negative eigenvalue. This is realized for a classical latent class model with one latent class. That is, the approximation is never exact, but will be better the closer the latent tree is to a latent class model with one latent class.

To conclude, item groups arise because multiple items connect to the same skill, and the 2PL arises from an approximation to the higher level skill structure. These results provide a solid foundation for the Dyad-4PL. 


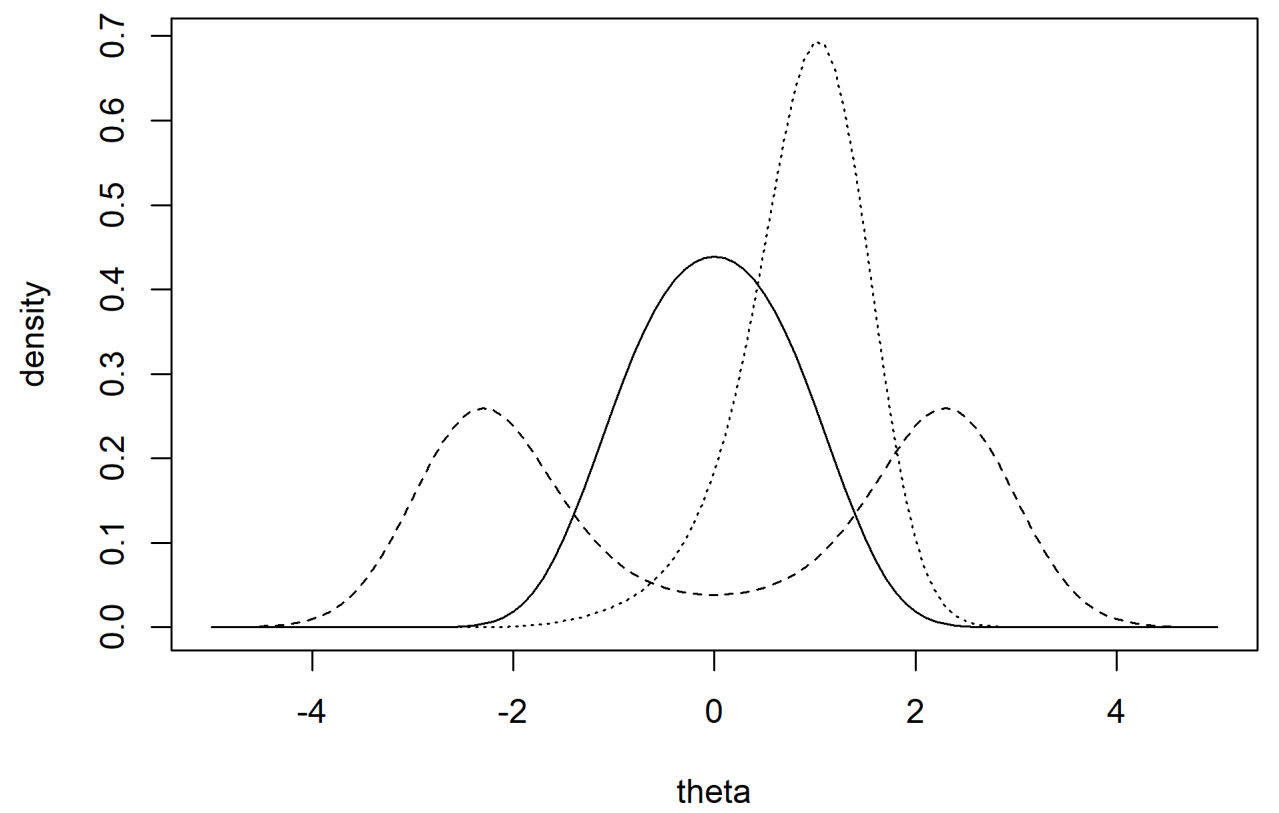

Figure 2. Distribution of the latent variable in Equation 10 for different magnitudes of the eigenvalue for a rank one approximation.

\section{Identifiability revisited}

For the general binary graphical model (i.e., Equation 4 where the matrix $\boldsymbol{\Sigma}$ could be any symmetric matrix), it is known that all parameters are identifiable from $\mathbf{W}$. For a graphical tree model this is particularly easy to see since the parameters are nothing but the joint distributions of neighboring nodes (see Equation 5).

We are interested, however, in parameter identifiability from the marginal distribution of the leaf nodes in a latent tree model. We start with some obvious necessary conditions and then show that, jointly, these are also sufficient. First, it is clear that any augmented variable in $p(\mathbf{y}, \mathbf{w})$ that only has two neighbors can be eliminated without changing the distribution of interest $p(\mathbf{y})$. In the left panel of Figure 1 , for example, node $W_{6}$ only has two neighbors $\left(Y_{1}\right.$ and $\left.W_{10}\right)$, and hence only figures in one equation:

$$
\boldsymbol{\pi}_{1 \mid 10}=\boldsymbol{\pi}_{1 \mid 6} \boldsymbol{\pi}_{6 \mid 10}
$$

with two unknowns. This system is clearly underdetermined, and the parameters on the right side are not identifiable. Formally, we integrate out augmented variables which have only two neighbors. Figure 1 illustrates the result of this elimination process. The left and middle panels in Figure 1 imply the exact same distribution for the observed variables, but the model on the left is not identifiable. Second, variables that are neighbors can not be independent of one another; otherwise, our tree would not be a tree, but rather a forest. 


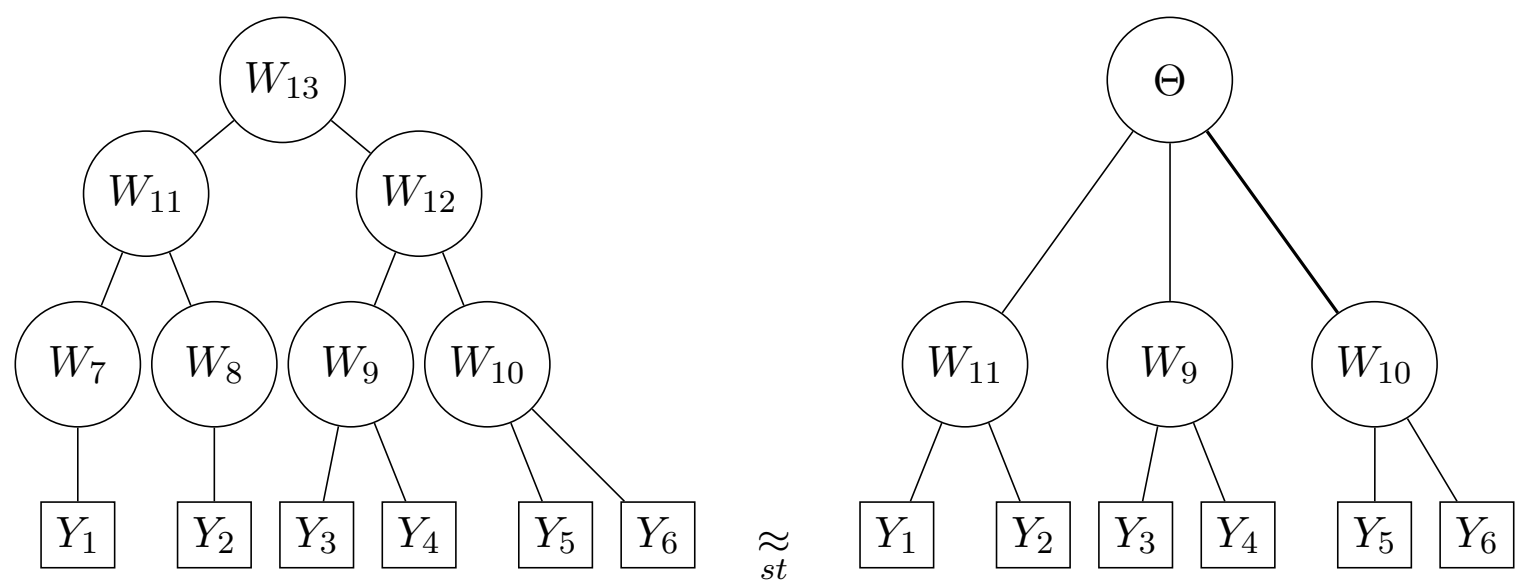

Figure 3. Unidentified latent tree model (left), and its approximate identified Dyad-4PL (right)

Third, neighbors can not be perfectly dependent (i.e., equal with probability one), in which case there would obviously be just one node rather than two.

The proof that these three conditions are not only necessary but also sufficient goes back at least to Pearl (1988). We offer a simple yet instructive proof. We pick two observed nodes $\left(Y_{1}\right.$ and $\left.Y_{2}\right)$ that are siblings, and an arbitrary third observed node $Y_{3}$. We integrate out (from the leaves up) all nodes that are not on the paths connecting these three nodes, as well as all remaining augmented nodes with only two neighbours. This process necessarily leaves us with a latent class model in which the three observed variables are connected via the same augmented node. As this is an identified model, taking our three conditions into account, so are the parameters for the two sibling nodes. This shows that the joint distribution of $Y_{1}, Y_{2}$, and $W_{10}$ is identified. This obviously holds for all dyads. If there are two different joint distributions for the augmented variables to which observed variables are attached, then the implied joint distributions of the observed variables are different as well. That is, the distribution of the augmented variables to which the observed ones are attached is identifiable. We then repeat the same exercise to identify the next level in the tree. We conclude that for a given tree, in which each non-leaf node has at least three neighbours, all parameters are identifiable, provided no two nodes are independent nor perfectly dependent.

The attentive reader may have noticed that the right hand side panel of Figure 1 is actually not a Dyad-4PL as node $Y_{5}$ does not belong to any dyad. Figure 3 shows a latent tree model and the approximate Dyad-4PL that goes with it. The same approach we used for the latent tree model also works for establishing identifiability of $p(\mathbf{y} \mid \mathbf{w})$ in the Dyad-4PL. As $p(\mathbf{w})$ is a marginal $2 \mathrm{PL}$, its identifiability conditions are well known. The same reasoning applies to any IRT model one wants to use for $p(\mathbf{w} \mid \theta)$, including the $2 \mathrm{PNO}$.

So far, we have dealt with parameter identifiability, assuming that the structure of the tree was known. We now turn to identifying the structure of the tree itself. For the purpose of this paper, we focus on recovering the grouping of items which is at the heart of the Dyad-4PL. This rests on a characterization of what it means for two nodes to be siblings. 
Assume that nodes 3 and 4 are siblings via the path 3-9-4 in Figure 3. Then, for a third node $j$ (where we spell out the equation for $j=5$ ):

$$
\begin{aligned}
& \boldsymbol{\pi}_{35}=\boldsymbol{\pi}_{3 \mid 9} \boldsymbol{\pi}_{9 \mid 12} \boldsymbol{\pi}_{12 \mid 10} \boldsymbol{\pi}_{10 \mid 5} \operatorname{diag}\left(\boldsymbol{\pi}_{5}\right), \text { and } \\
& \boldsymbol{\pi}_{45}=\boldsymbol{\pi}_{4 \mid 9} \boldsymbol{\pi}_{9 \mid 12} \boldsymbol{\pi}_{12 \mid 10} \boldsymbol{\pi}_{10 \mid 5} \operatorname{diag}\left(\boldsymbol{\pi}_{5}\right)
\end{aligned}
$$

which means we can solve the equations (for any $j$ ) to obtain

$$
\forall j: \boldsymbol{\pi}_{3 j} \boldsymbol{\pi}_{4 j}^{-1}=\boldsymbol{\pi}_{3 \mid 9} \boldsymbol{\pi}_{4 \mid 9}^{-1}
$$

if and only if nodes 3 and 4 are siblings (in an identifiable latent tree model). We've put in a definite path in the expressions above between nodes 3 and 4 and node $j$, but as is clear from the expressions the essential feature of siblings is that the right-hand side equations can only differ in $\boldsymbol{\pi}_{3 \mid 9}$ and $\boldsymbol{\pi}_{4 \mid 9}$. As Equation 14 involves matrix inverses, these need to exist. However, as before this entails that no observed variable is independent of its augmented variable, and hence we deal with a tree and not a forest. Note that if the right-hand side of Equation 14 equals $\boldsymbol{\pi}_{3 \mid 4}$, then nodes 3 and 4 are neighbors.

It is clearly sufficient for Equation 14 to hold for bivariate distributions of observed variables in the left-hand side, and hence we can identify whether or not any two observed variables are siblings, which is all we need for the Dyad-4PL. If, as in the middle panel of Figure 1, you are left with a node $\left(Y_{5}\right)$ without siblings, you need to infer more of the structure of the latent tree. Fortunately, this proceeds along the same lines, and leads to the conclusion that $Y_{5}$ is a sibling of $W_{11}$, and in fact, the structure of the whole tree can be inferred.

\section{Implications for psychological measurement}

\section{Test construction and analysis}

Both summative and formative tests are typically based on a curriculum that is hierarchically organized. Mathematics, for example, is subdivided into analysis, algebra, and geometry, with algebra being divided into linear and quadratic equations, quadratic equations involving those with one and those with two real zeroes, etc.

A summative test typically aims for broad coverage of the hierarchical framework, with few items (often just one) tagged to each lowest level skill. A formative test, on the contrary, aims to cover a narrow part of the framework with many items tagged to a handful of skills. With one item per skill, our results on identifiability suggest that summative tests may be in trouble. Moreover, connecting formative and summative tests seems challenging. Neither however is true.

For any non-identifiable latent tree model, we can always construct an equivalent model that is identifiable. The latter can be approximated by an identifiable Dyad-4PL. The parameters of both models are connected through Equation 7 . What does that mean? Suppose a test contains one item $\left(Y_{1}\right)$ on quadratic equations with one real root $\left(W_{7}\right)$, and one $\left(Y_{2}\right)$ on quadratic equations with two real roots $\left(W_{8}\right)$. In this situation we can identify the joint distribution of each of the items and the higher-order latent skill $\left(W_{11}\right)$, but not the joint distributions with the lower-order skills. If, in a second version of the test, we include the same two items, and add one of each type, we can all of a sudden identify the 
joint distribution of each item and a lower order skill. In a latent tree model this does not pose a problem as we know how the parameters from both statistical models relate to one another. In the corresponding approximate Dyad-4PL this is not the case, since there is no notion of higher order skills. One can only register that the parameter is different in both tests, but the model is not rich enough to offer a (testable) explanation.

The relation between a summative test which targets higher level skills and a formative test which, say, targets lower level skills of just one or two of these higher level skills is governed by Equation 7. If you wish to report on a skill you need to ensure that you design the test such that it is identifiable. Fortunately, it is not difficult to construct the implied identified latent tree model to check this.

\section{The interpretation of parameters}

There is a long tradition in psychometrics to attach person and item indices to parameters, and refer to them as person and item parameters, respectively. That this is not without problems can be illustrated in the context of latent tree and Dyad-4PL models.

We get to see some of these problems when we look back at the example with two types of quadratic equations that was mentioned in the previous section. In the first test (with one of each type) the item parameters are the left hand sides of

$$
\begin{aligned}
& \boldsymbol{\pi}_{1 \mid 11}=\boldsymbol{\pi}_{7 \mid 11} \boldsymbol{\pi}_{1 \mid 7} \\
& \boldsymbol{\pi}_{2 \mid 11}=\boldsymbol{\pi}_{8 \mid 11} \boldsymbol{\pi}_{2 \mid 8}
\end{aligned}
$$

whereas in the second test (with two of each type) they are $\boldsymbol{\pi}_{1 \mid 7}$, and $\boldsymbol{\pi}_{2 \mid 8}$. First, these are not the same, but more importantly in the former case the item parameter is a function of parameters that characterize the population of test takers. Second, even in the latter case we can not rule out that quadratic equations with one real root can be split into even more fine grained classes, such that the same reasoning applies.

This clearly also impacts on the interpretation of item parameters as guessing and slipping parameters. In both tests these would be different, for the same item. For the latent tree model, the situation is clear, for the approximate Dyad-4PL things are more complicated. For the Dyad-4PL it is clear however, that items that form a dyad in one test, need no longer form a dyad in another test, and that an item that is connected with one augmented variable in one test may be connected to a different augmented variable in another test.

Similarly for the item and item group parameters of the $2 \mathrm{PL}$, in respectively the $4 \mathrm{PL}$ and the Dyad-4PL, when considered an approximation to a latent tree model, the difficulty and discrimination parameters are not properties of the item, or item group. Rather these derive from a joint distribution of skills, in a population of test takers, which are identifiable from responses to a particular test.

Further, assuming all non-zero elements of $\boldsymbol{\Sigma}$ to be equal, we find that $W_{10}$ has a higher discrimination than $W_{9}$ and $W_{11}$ in the approximate Dyad-4PL, which we represented with a thicker edge in Figure 1. That is, even though all parameters are the same in the latent tree model, the resulting discrimination parameters in the approximate Dyad-4PL are not.

Finally, everyone knows this kid who says "I've never understood quadratic equations, but I'm good at geometry though." If this kid were to take two tests containing both 
quadratic equations and geometry items what are we to expect? We interpret the statement as meaning that, for an individual, the augmented variables stay the same (or vary slowly) over time. This reflects that students don't all of a sudden get better at everything (increase in $\theta$ ), nor do items all of a sudden get easier for everyone (decrease in $\beta_{i}$ ). In this situation, both the latent tree model and the Dyad-4PL lead us to expect the kid to do poorly on quadratic equations (again), and better on geometry. At the level of a skill, or grouping, test-retest reliability is high(er). For the $4 \mathrm{PL}$ this would not be the case. This leads to a directly testable prediction: test-retest reliability is underestimated in a $4 \mathrm{PL}$ if we take the latent tree or Dyad-4PL model seriously.

\section{Discussion}

We have introduced a latent tree model that implies the Dyad-4PL (and Dyad-4PNO) as an approximation. This latent tree model gives insight into where the grouping of items in the Dyad-4PL comes from, and what the nature of ability in this model is (or could be). Furthermore, the issue of identifiability of both groupings and parameters is greatly simplified. The latent tree model itself can exactly be represented as a MIRT model (see Equations 9 and 10) and, if one assumes the tree model only for the augmented variables, as a Dyad-MIRT model.

The latent tree perspective also sheds light on the identifiability of the $3 \mathrm{Pl}$ and $4 \mathrm{PL}$ model. Suppose that no two observed variables in a latent tree model have the same augmented variable. Clearly, the parameters are not identifiable, but we get an approximate 4PL. This approximate 4PL need not be identifiable, but using the same elimination process that was used to prove the necessary and sufficient conditions for the identifiability of a latent tree model, we obtain an identifiable Dyad-4PL.

Driven by a desire to make more meaningful interpretations and inferences, researchers in psychometrics often succumb to the temptation to interpret model parameters as concrete person and item characteristics rather than abstractions and functions of these respective populations. This error, known as the reification fallacy, is not made without consequence, as we've illustrated. Note that reification is much stronger than merely giving parameters an item index, and referring to them as item parameters, ignoring that they only have meaning relative to a given test and a given population of test takers. That does not imply that it is not possible to relate item parameters across tests and populations in a meaningful way.

We started this paper with the question "why does the Dyad-4PNO model of Kern and Culpepper (2020) fit real data?". We can ask the same question about the latent tree model. Is it a realistic model? Yes, in the sense that it fits closely to the (hierarchical) frameworks that guide the development of assessments. But overall, the answer is probably negative as the model lacks some key components. First, some items tap into more than just one skill. When, for example, a mathematical problem is posed as a story, it may depend on reading comprehension for its solution. A science problem may require critical thinking skills. Cognitive diagnosis models were developed to deal with exactly this situation. Second, mastering quadratic equations when you haven't yet mastered linear equations seems like an unlikely state of affairs. Doignon and Falmagne (2012) introduced knowledge spaces to take account of the pre-requisite relations between skills. Deonovic, Bechger, and Maris (2020) fuse the latent tree model with cognitive diagnosis models and knowledge spaces, into 
a model that is both more realistic and more useful for supporting (personalized) learning and assessment than either of its ingredients on its own.

Even though we worked through the details for a latent tree model, as it is appropriate in an educational measurement context, different options exist for different substantive areas (e.g., personality). The key ingredient to obtaining the Dyad-4PL as an approximation is that observed variables are leaf nodes in some graphical model, where the quality of the approximation depends on how close the adjacency matrix of the latent part of the graphical model is to a rank one matrix.

To return to the Dyad-4PL proper, we see another fruitful area of application. Even though the model is originally framed for multiple choice questions, it equally applies to open ended questions that are coded (right/wrong) by human coders. If we interpret the augmented variables as representing the true quality of a response, and the observed variables as codes assigned by human coders we get a model similar to those of Patz, Junker, Johnson, and Mariano (2002) and Verhelst and Verstralen (2001). It is fairly easy to impose constraints on the guessing and slipping parameters to make them coder specific, or item specific, and to extend the model to deal with polytomous codes.

\section{References}

Barton, M. A., \& Lord, F. M. (1981). An upper asymptote for the three-parameter logistic itemresponse model. ETS Research Report Series, 1981 (1), i-8.

Camara, W., O'connor, R., Mattern, K., \& Hanson, M. A. (2015). Beyond academics: A holistic framework for enhancing education and workplace success. ACT Research Report Series. 2015 (4). ACT, Inc..

De La Torre, J., \& Douglas, J. A. (2004). Higher-order latent trait models for cognitive diagnosis. Psychometrika, 69(3), 333-353.

Deonovic, B., Bechger, T., \& Maris, G. (2020, Sep). The Ising on the tree: The Master model for learning, assessment, and navigation. PsyArXiv. Retrieved from psyarxiv.com/t65wa doi: 10.31234/osf.io/t65wa

Doignon, J.-P., \& Falmagne, J.-C. (2012). Knowledge spaces. Springer Science \& Business Media.

Eckart, C., \& Young, G. (1936). The approximation of one matrix by another of lower rank. Psychometrika, 1(3), 211-218.

Epskamp, S., Maris, G. K., Waldorp, L. J., \& Borsboom, D. (2016). Network psychometrics. arXiv preprint arXiv:1609.02818.

Gu, Y., \& Xu, G. (2018). Sufficient and necessary conditions for the identifiability of the Q-matrix. arXiv preprint arXiv:1810.03819.

Kern, J. L., \& Culpepper, S. A. (2020). A restricted four-parameter IRT model: The dyad fourparameter normal ogive (dyad-4PNO) model. Psychometrika, 1-25.

Kruis, J., \& Maris, G. (2016). Three representations of the Ising model. Scientific reports, 6, 34175.

Maris, G., \& Bechger, T. (2009). On interpreting the model parameters for the three parameter logistic model. Measurement, 7(2), 75-88.

Marsman, M., Borsboom, D., Kruis, J., Epskamp, S., van Bork, R., Waldorp, L., ... Maris, G. (2018). An introduction to network psychometrics: Relating Ising network models to item response theory models. Multivariate behavioral research, 53(1), 15-35.

Marsman, M., Maris, G., Bechger, T., \& Glas, C. (2015). Bayesian inference for low-rank Ising networks. Scientific reports, 5(1), 1-7.

Patz, R. J., Junker, B. W., Johnson, M. S., \& Mariano, L. T. (2002). The hierarchical rater model for rated test items and its application to large-scale educational assessment data. Journal of Educational and Behavioral Statistics, 27(4), 341-384. 
Pearl, J. (1988). Probabilistic reasoning in intelligent systems. morgan kauffmann san mateo. Verhelst, N. D., \& Verstralen, H. H. (2001). An irt model for multiple raters. In Essays on item response theory (pp. 89-108). Springer.

Wainwright, M. J., \& Jordan, M. I. (2008). Graphical models, exponential families, and variational inference. Now Publishers Inc. 